\title{
Article \\ Super Vertex (Edge)-Connectivity of Varietal Hypercube
}

\author{
Zhecheng $\mathrm{Yu}^{1,2}$, Liqiong $\mathrm{Xu}{ }^{1,2, * \mathbb{D}}$, Shanshan Yin ${ }^{1,2}$ and Litao Guo ${ }^{3}$ \\ 1 School of Science, Jimei University, Xiamen 361021, China; 202011701007@jmu.edu.cn (Z.Y.); \\ 201911701009@jmu.edu.cn (S.Y.) \\ 2 Digital Fujian Big Data Modeling and Intelligent Computing Institute, Xiamen 361021, China \\ 3 Department of Mathematics, Xiamen University of Technology, Xiamen 361024, China; guolitao@xmut.edu.cn \\ * Correspondence: xuliqiong@jmu.edu.cn
}

check for

updates

Citation: Yu, Z.; Xu, L.; Yin, S.; Guo, L. Super Vertex (Edge)-Connectivity of Varietal Hypercube. Symmetry 2022, 14, 304. https://doi.org/ $10.3390 /$ sym 14020304

Academic Editors: Guifu Su and Junfeng Du

Received: 25 December 2021

Accepted: 28 January 2022

Published: 2 February 2022

Publisher's Note: MDPI stays neutral with regard to jurisdictional claims in published maps and institutional affiliations.

Copyright: (C) 2022 by the authors. Licensee MDPI, Basel, Switzerland. This article is an open access article distributed under the terms and conditions of the Creative Commons Attribution (CC BY) license (https:// creativecommons.org/licenses/by/ $4.0 /)$.

\begin{abstract}
The reliability measure of networks is of significant importance to the design and maintenance of networks. Based on connectivity, many refined quantitative indicators for the reliability of network systems have been introduced. The super vertex edge-connectivity and cyclic edgeconnectivity, as important parameters to evaluate the robustness of networks, are explored extensively. As a variant of the hypercube $Q_{n}$, the varietal hypercube $V Q_{n}$ has better properties than $Q_{n}$ with the same number of edges and vertices. Wang and $\mathrm{Xu}$ have proved that $V Q_{n}$ is super vertex-connected for $n \geq 1$ and is also super edge-connected if $n \neq 2$. In this paper, we use another method to prove these results. Moreover, we also obtain the super restricted connectivity and the cyclic edge-connectivity of the varietal hypercube $V Q_{n}$.
\end{abstract}

Keywords: reliability; super vertex (edge)-connectivity; cyclic edge-connectivity; vertex-transitivity; edge-transitivity; varietal hypercube

\section{Introduction}

In the era of internet-of-things (IoT), wireless sensor networks (WSNs) are utilised in many applications, including smart grids (SGs) [1]. When the number of sensors continues to increase, faulty nodes and faulty links are inevitable in wireless sensor networks, which undoubtedly increase the difficulty of network reliability assessment and will adversely affect its fault tolerance and reliability.

Hence, to the design and maintenance purpose of networks, appropriate measures of reliability should be found. Because the topology of network system can usually be represented by a connected graph $G=(V(G), E(G))$, where $V(G)$ and $E(G)$ can be represented as the set of processors and the set of communication links between processors, respectively. Connectivity $\kappa(G)$ and edge-connectivity $\lambda(G)$ represent the maximum number of faulty vertices and the maximum number of faulty edges that an interconnected network can tolerate in normal communication, respectively. Obviously, the higher the (edge-) connectivity is, the more reliable an interconnection network is [2]. As is known, $\kappa(G) \leq \lambda(G) \leq \delta(G)$, where $\delta(G)$ is the minimum degree of $G$. Boesch and Tindell [3] introduced the concepts of super connectivity and super edge- connectivity. A connected graph $G$ is super connected (resp., edge-connected, in short super- $\kappa$ (resp., super- $\lambda$, ) if any minimum vertex-cut (resp., edge-cut) isolates a vertex of $G$.

However, the biggest disadvantage of connectivity and edge-connectivity in reliability evaluation and fault-tolerant performance of networks is that they all default to a vertex where all adjacent vertices or adjacent edges fail at the same time, which rarely happens in the real world. To overcome such shortcomings, several new concepts on the (edge-) connectivity of graphs, which are called conditional (edge-) connectivity, were proposed by Harary [4]. Among them are restricted (edge-)connectivity and cyclic edge-connectivity.

Esfahanian and Hakimi proposed the concept of restricted connectivity [5]. For a given non-negative integer $h$, a vertex-set (resp., edge-set) $F$ is an $h$-restricted vertex-cut (resp., 
edge-cut) of a connected graph $G$ if $G-F$ is disconnected, and each component of $G-F$ contains at least $h+1$ vertices. The minimum size of all $h$-restricted vertex-cuts (resp., edge-cuts), denoted by $\kappa^{(h)}(G)$ (resp., $\lambda^{(h)}(G)$ ), is called $h$-restricted connectivity (resp., edge-connectivity) of the graph $G$. In the exceptional cases, $G$ is called super restricted vertex (edge)-connected and denoted by super- $\kappa^{\prime}$ (resp., super- $\lambda^{\prime}$ ), if every minimum restricted edge cut isolates one component of order 2 . The $h$-restricted (edge)-connectivity and super- $\kappa^{\prime}$ (resp., super- $\lambda^{\prime}$ ) graph for some classic interconnection networks have been investigated recently. For instance, Li et al. [3] determine that the $h$-restricted connectivity of the arrangement graph $A_{n, k}$ is $n(n-1) / 2$ for $h$ is odd and $h=n-3$, otherwise the $h$-restricted connectivity of the arrangement graph $A_{n, k}$ is $(h+2)(n-2)-\left\lfloor h^{2} / 2+h\right\rfloor$ for $k=2$ and $0 \leq h \leq n-2$. Chen et al. [6] proved that the hypercube, the twisted cube, the cross cube, the Möbius cube, and the locally twisted cube are super connected and super edge-connected. Futhermore, $\mathrm{Xu}$ et al. [7] obtained super edge-connectivity, and the restricted (edge-) connectivity of the hypercube, the twisted cube, the cross cube, Möbius cube, and the locally twisted cube is $2 n-2$ for $n \geq 3$. Zhou et al. [8] obtained that the balanced hypercube $B H_{n}$ is super- $\lambda^{\prime}$ but not super- $\kappa^{\prime}$ for $n \geq 2$.

In 1978, Bollobás indicated all multigraphs without two vertex-disjoint cycles [9]. So, it is necessary to further study the cyclically separable graphs. An edge set $S$ is a cyclic edgecut if $G-S$ is disconnected and at least two of its components contain cycles. Obviously, a graph $G$ has a cyclic edge-cut if and only if it has two vertex-disjoint cycles and is a cyclically separable graphs. For a cyclically separable graph $G$, the cyclic edge-connectivity of $G$, denoted by $\lambda_{c}(G)$, is defined as the cardinality of the minimum cyclic edge-cut of $G$ [10]. Let $\omega(X)$ be the number of edges with one end in $X$ and another end in $V(G)-X$, and $\xi(G)=\min \{\omega(X) \mid X$ induces a shortest cycle in $G\}$. A cyclically separable graph $G$ with $\lambda_{c}(G)=\xi(G)$ is called cyclically optimal. Moreover, if removing any minimum cyclic edge-cut of the graph $G$ results in the shortest cycle in a component of the graph $G$, then the graph $G$ is said to be super cyclically edge-connected [11], in short, super- $\lambda_{c}$. There are some results of the super cyclically edge-connectivity for some classes of the graphs. For example, Zhang [12] showed that the cyclic edge-connectivity of a strongly regular connected graph $G$ (not $K_{3,3}$ ) of degree $k \geq 3$ with girth $g$ is equal to $(k-2) g$, where $g=3,4$ or 5. Wang and Zhang [13] showed that any $k$-regular vertex-transitive connected graph with $k \geq 4$ and girth of at least 5 is cyclically optimal, and any edgetransitive connected graph with minimum degree of at least 4 and order of at least 6 is cyclically optimal.

Hypercube $Q_{n}$, one of the most important and attractive network topologies so far, is widely used due to its excellent characteristics. In 1994, Cheng and Chuang designed a network topology capable of obtaining an interconnection network that is both efficient and cost-effective, this is, the varietal hypercube $V Q_{n}$. In recent years, $V Q_{n}$ has received considerable attention and many of its properties have been studied. For example, Zhou [14] showed that $V Q_{n}$ is a Cayley graph. Wang et al. [15] also determined the full automorphism group of the varietal hypercube $V Q_{n}$. More relevant results on the varietal hypercube $V Q_{n}$ are shown in [16-20].

Our work in this paper concerns the $n$-dimensional varietal hypercube $V Q_{n}$. We use another method to prove that $V Q_{n}$ is super vertex-connected for any $n \geq 1$ and super edge-connected for $n \neq 2$. We also obtain that $V Q_{n}$ is super- $\lambda_{c}$ for $n \geq 3$, super- $\lambda^{\prime}$ for $n \geq 3$ and $n \neq 4$, and the cyclic edge-connectivity of $V Q_{n}$ is $4(n-2)$ for $n \geq 3$.

The paper is organized as follows. In Section 2, we give some propositions related to the vertex-transitive and edge-transitive graph. In Section 3, we introduce the necessary definitions and notations. In Section 4 , we use the symmetry properties of the $n$-dimensional varietal hypercube to discuss some reliability measures of the $n$-dimensional varietal hypercube. In Section 5, we add some concluding remarks. 


\section{Related Works}

We use a number of known results about some propositions of the vertex-transitive and edge-transitive graphs in the following.

Proposition $1([21,22])$. Let $G$ be a connected graph that is both vertex-transitive and edgetransitive. Then $\kappa(G)=\delta(G)$, and moreover, $G$ is not super- $\kappa$ if and only if $G \cong C_{n} \circ m K_{1}$ $(n \geq 6)$ or $L\left(Q_{3}\right) \circ m K_{1}$, where $L\left(Q_{3}\right)$ is the line graph of the three dimensional hypercube $Q_{3}$.

Proposition 2 ([23-27]). Let $G$ be an edge-transitive $k$-regular graph where $k \geq 3$. Then

(1) $G$ is super- $\lambda$.

(2) $\lambda^{\prime}(G)=2 k-2$.

(3) If $G$ is not isomorphic to $K_{4}, K_{5}$ or $K_{3,3}$, then $\lambda_{c}(G)=g(k-2)$, where $g$ is the girth of $G$.

(4) $G$ is not super- $\lambda^{\prime}$ if and only if $G$ is isomorphic to the three dimensional hypercube $Q_{3}$ or to an edge-transitive 4-regular graph of girth 4 .

Let $\mathbb{Z}_{n}$ be denoted the residues modulo $n$ for any positive integer $n$. We denote by $\mathcal{G}_{16}$ a graph with vertex set $\left\{a_{i}, b_{i} \mid i \in \mathbb{Z}_{8}\right\}$, and for each $i \in \mathbb{Z}_{8}$, if $i$ is odd then $a_{i} b_{i+j} \in E\left(\mathcal{G}_{16}\right)$ with $0 \leq j \leq 2$ and $b_{i} a_{i+j} \in E\left(\mathcal{G}_{16}\right)$ with $0 \leq j \leq 2$, and if $i$ is even then $a_{i} b_{i+j} \in E\left(\mathcal{G}_{16}\right)$ with $0 \leq j \leq 3$ and $b_{i} a_{i+j} \in E\left(\mathcal{G}_{16}\right)$ with $0 \leq j \leq 3$. If $n \geq 3$ and $1 \leq t<n / 2$, the generalized petersen graph $P(n, t)$ is a graph with vertex set $\left\{x_{i}, y_{i} \mid i \in \mathbb{Z}_{n}\right\}$ and edge set $\left\{\left\{x_{i}, x_{i+1}\right\},\left\{x_{i}, y_{i}\right\},\left\{y_{i}, y_{i+t}\right\} \mid i \in \mathbb{Z}_{n}\right\}$. For a 4-regular graph $H$, the subdivided double $\mathcal{D}(H)$ of $H$ is the bipartite graph with the vertex set $\left(V(H) \times \mathbb{Z}_{2}\right) \cup E(H)$ and an edge between vertices $(v, i) \in V(H) \times \mathbb{Z}_{2}$ and $e \in E(H)$ whenever $v$ is incident with e in $H$, where $\left(V(H) \times \mathbb{Z}_{2}\right)=\{(u, 0),(u, 1) \mid u \in V(H)\}$. The graph $G-m K_{2}$ is obtained by removing $m$ vertex disjoint edges from $G$.

In [28], Zhou and Feng have classified all $\lambda_{c}$-optimal nonsuper- $\lambda_{c}$ edge-transitive regular graphs and obtained some properties of $\lambda_{c}$-optimal nonsuper- $\lambda_{c}$ edge-transitive regular graphs.

Proposition 3 ([28]). Let $G$ be an edge-transitive $k$-regular graph of girth $g$ where $k \geq 3$. Suppose that $G$ is not isomorphic to $K_{4}, K_{5}$, or $K_{3,3}$. Then, $G$ is not super- $\lambda_{c}$ if and only if at least one of the following holds.

(1) $g=6, k=3$ and $G \cong P(10,7)$ or $P(8,3)$.

(2) $g=4$ and $k=4, G$ is isomorphic to $Q_{4}$ (the 4-cube), $K_{5.5}-5 K_{2}, C_{n} \circ 2 K_{1}(n \geq 4)$ or $\mathcal{D}(H)$ for some arc-transitive 4-regular graph $H$.

(3) $g=4, k=5$ and $G \cong K_{6.6}-6 K_{2}$.

(4) $g=4, k=6$ and $G \cong \mathcal{G}_{16}$.

(5) $g=3, k=6$ and $G \cong K_{8}-4 K_{2}$ or $G$ is isomorphic to the line graph of an arc-transitive 4-regular triangle-free graph.

\section{Preliminaries}

In this section, we give some definitions and notations. If definitions and notations are not defined here, we follow [29].

Let $A u t(G)$ be the automorphism group of the graph $G$. We say that $G$ is vertextransitive if for any two vertices $u, v \in V(G)$ there exists an automorphism $\pi \in \operatorname{Aut}(G)$ such that $\pi(u)=v$. If for any pair of edges (resp. arcs), there exists an automorphism that transforms one into the other, then $G$ is edge-transitive (resp. arc-transitive) graph. Clearly, if a graph is arc-transitive, then it must be vertex-transitive and edge-transitive. Let $u$ and $v$ be two vertices of $G$, and the length of a shortest path is called the distance and denoted by $d_{G}(u, v)$. The maximum distance between any pair of vertices of a connected graph $G$ is called the diameter of the graph $G$ and denoted by $\operatorname{diam}(G)$.

Suppose that $G$ and $H$ are two graphs, the lexicographic product $G \circ H$ is defined as the graph with vertex set $V(G) \times V(H)$, two vertices $\left(x_{1}, y_{1}\right)$ and $\left(x_{2}, y_{2}\right)$ are adjacent if and only if either $x_{1} x_{2} \in E(G)$, or $x_{1}=x_{2}$ and $y_{1} y_{2} \in E(H)$. The graph showed in Figure 1 is $C_{6} \circ 2 K_{1}$. 


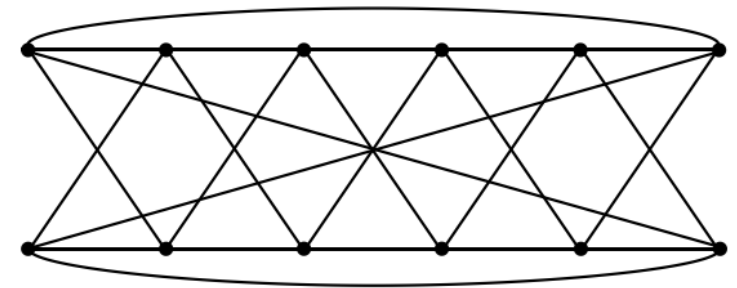

Figure 1. The lexicographic product of $C_{6} \circ 2 K_{1}$.

Let $n$ be a positive integer. The definitions of the $n$-dimensional hypercube $Q_{n}$ and the $n$-dimensional varietal hypercube $V Q_{n}$ are stated as follows.

Definition 1 ([30]). The n-dimensional hypercube is a connected graph with $2^{n}$ vertices and denoted by $Q_{n}$. The vertex set $V\left(Q_{n}\right)=\left\{x_{1} x_{2} \cdots x_{n}: x_{i}=0\right.$ or $\left.1,1 \leq i \leq n\right\}$. Two vertices $u=u_{1} u_{2} \cdots u_{n}$ and $v=v_{1} v_{2} \cdots v_{n}$ in $Q_{n}$ are adjacent if and only if they differ in exact one position.

Definition 2 ([17]). The n-dimensional varietal hypercube, denoted by $V Q_{n}$, has $2^{n}$ vertices, each labeled by an $n$-bit binary string and $V\left(V Q_{n}\right)=\left\{x_{n} x_{n-1} \cdots x_{2} x_{1}: x_{i}=0\right.$ or $\left.1, i=1,2, \ldots, n\right\}$. $V Q_{1}$ is a complete graph $K_{2}$ of two vertices labeled with 0 and 1 , respectively. For $n \geq 2$, $V Q_{n}$ can be recursively constructed from two copies of $V Q_{n-1}$, denoted by $V Q_{n-1}^{0}$ and $V Q_{n-1}^{1}$, and by adding $2^{n-1}$ edges between $V Q_{n-1}^{0}$ and $V Q_{n-1}^{1}$, where $V\left(V Q_{n-1}^{0}\right)=\left\{0 x_{n-1} \cdots x_{2} x_{1}\right.$ : $x_{i}=0$ or $\left.1, i=1,2, \ldots, n-1\right\}, V\left(V Q_{n-1}^{1}\right)=\left\{1 x_{n-1} \cdots x_{2} x_{1}: x_{i}=0\right.$ or $1, i=$ $1,2, \ldots, n-1\}$. The vertex $x=0 x_{n-1} x_{n-2} x_{n-3} \cdots x_{2} x_{1} \in V\left(V Q_{n-1}^{0}\right)$ is adjacent to the vertex $y=1 y_{n-1} y_{n-2} y_{n-3} \ldots y_{2} y_{1} \in V\left(V Q_{n-1}^{1}\right)$ if and only if

$$
x_{n-1} x_{n-2} x_{n-3} \cdots x_{2} x_{1}=y_{n-1} y_{n-2} y_{n-3} \cdots y_{2} y_{1} \text { if } 3 \nmid n, \text { or }
$$

$x_{n-3} \cdots x_{2} x_{1}=y_{n-3} \cdots y_{2} y_{1}$ and $\left(x_{n-1} x_{n-2}, y_{n-1} y_{n-2}\right) \in\{(00,00),(01,01),(10,11),(11,10)\}$ if $3 \mid n$.

Obviously, $V Q_{n}$ is an n-regular and its girth is 4 . Moreover, it contains circles of length 5 when $n \geq 3[18,19]$. The varietal hypercubes $V Q_{1}, V Q_{2}$, and $V Q_{3}$ are illustrated in Figure 2.

As a variant of the hypercube, the $n$-dimensional varietal hypercubes $V Q_{n}$, which has the same number of vertices and edges as $Q_{n}$, not only has the most ideal characteristics of $Q_{n}$, including some characteristics such as recursive structure, strong connectivity, and symmetry but also has a smaller diameter than $Q_{n}$, and its average distance is smaller than the hypercube [17].

Lemma 1 ([17]). $\operatorname{diam}\left(V Q_{n}\right)=\lceil 2 n / 3\rceil$ for $n \geq 3$.

Lemma 2 ([14]). $\kappa^{(2)}\left(V Q_{n}\right)>\kappa^{(1)}\left(V Q_{n}\right)$ for $n \geq 9$.

Lemma $3([15,20,31]) . V Q_{n}$ is vertex-transitive and edge-transitive.

Lemma 4 ([32]). Let $G$ and $H$ be two graphs. If $G$ is non-trivial, non-complete, and connected, then $\kappa(G \circ H)=\kappa(G)|V(H)|$.

Lemma 5 ([33]). Suppose $\left(x_{1}, y_{1}\right)$ and $\left(x_{2}, y_{2}\right)$ are two vertices of $G \circ H$. Then, the distance between $\left(x_{1}, y_{1}\right)$ and $\left(x_{2}, y_{2}\right)$ is

$$
d_{G \circ H}\left(\left(x_{1}, y_{1}\right),\left(x_{2}, y_{2}\right)\right)=\left\{\begin{array}{cl}
d_{G}\left(x_{1}, x_{2}\right) & \text { if } x_{1} \neq x_{2}, \\
d_{H}\left(y_{1}, y_{2}\right) & \text { if } x_{1}=x_{2} \text { and } d_{G}\left(x_{1}\right)=0, \\
\min \left\{d_{H}\left(y_{1}, y_{2}\right), 2\right\} & \text { if } x_{1}=x_{2} \text { and } d_{G}\left(x_{1}\right) \neq 0
\end{array}\right.
$$


From the above lemma, we can obtain that the diameter of $G \circ H$ is

$$
\operatorname{diam}(G \circ H)=\max \left\{d_{G \circ H}\left(\left(x_{1}, y_{1}\right),\left(x_{2}, y_{2}\right)\right):\left(x_{1}, y_{1}\right),\left(x_{2}, y_{2}\right) \in V(G) \times V(H)\right\}
$$
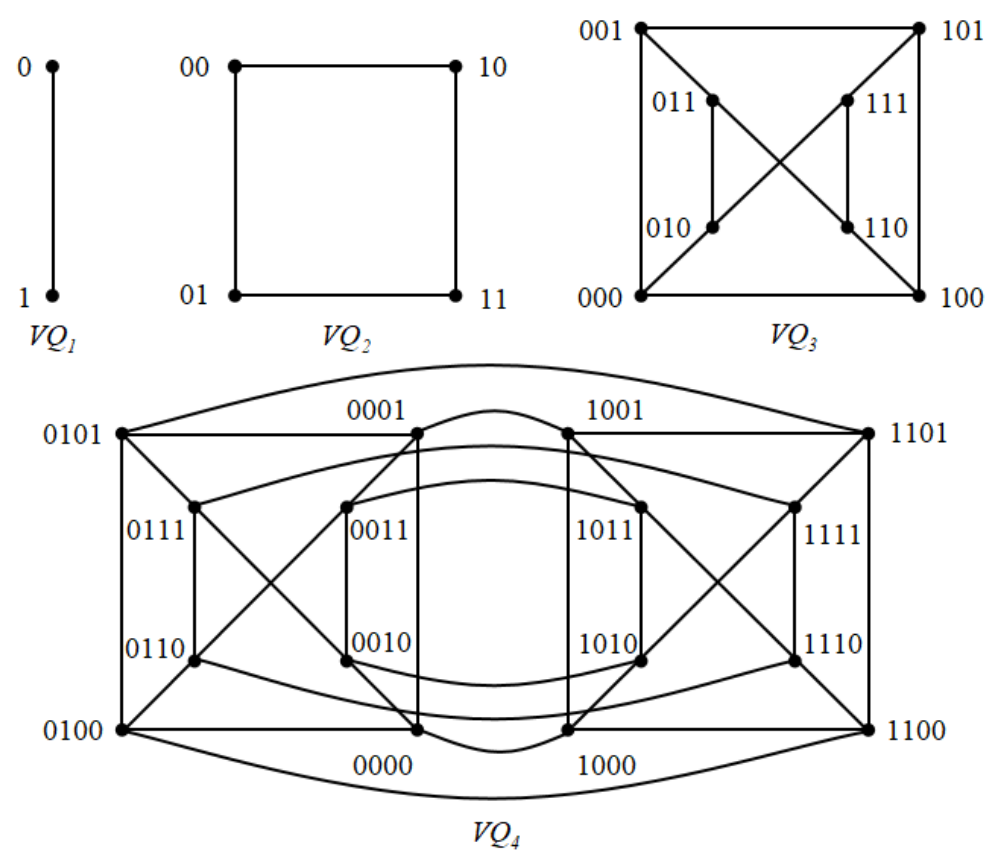

Figure 2. The varietal hypercubes $V Q_{1}, V Q_{2}, V Q_{3}$, and $V Q_{4}$.

\section{Reliability Evaluation of Varietal Hypercubes}

In this section, we will study some reliability measures in varietal hypercubes, such as restricted (vertex-)connectivity and cyclic connectivity. Although $\mathrm{Xu}$ et al. proved that $V Q_{n}$ is super vertex-connected for any $n \geq 1$ and super edge-connected for $n \neq 2$ in [19], we prove these results and obtain other results such as $V Q_{n}$ is super- $\lambda_{c}$ for $n \geq 3$, super- $\lambda^{\prime}$ for $n \geq 3$, and $n \neq 4$ through symmetric properties of $V Q_{n}$.

Theorem 1. $V Q_{n}$ is super $\kappa$ for $n \geq 1$.

Proof. If $n=1$, then $V Q_{n}$ is a complete graph $K_{2}$. Obviously, it is super- $\kappa$.

If $n=2$, then $V Q_{2}$ is cycle $C_{4}$, and clearly it is super- $\kappa$.

Assume that $n \geq 3$. Suppose to the contrary that $V Q_{n}$ is not super- $\kappa$. Since $V Q_{n}$ is both vertex-transitive and edge-transitive, it follows that $V Q_{n} \cong C_{l} \circ m K_{1}(l \geq 6)$ or $L\left(Q_{3}\right) \circ m K_{1}$ from Proposition 1.

First suppose that $V Q_{n} \cong C_{l} \circ m K_{1}(l \geq 6)$; we know that $\kappa\left(V Q_{n}\right)=n$ and $\kappa\left(C_{l} \circ\right.$ $\left.m K_{1}\right)=2 m$ by Lemma $4, n=2 m$ and $m>1$. Moreover, since $\left|V\left(C_{l} \circ m K_{1}\right)\right|=\left|V\left(V Q_{n}\right)\right|$, it follows that $2^{n}=m l$. Thus, $m$ and $l$ are even. According to Lemma 1 and Lemma 5 , we know that $\operatorname{diam}\left(V Q_{n}\right)=\lceil 2 n / 3\rceil$ and $\operatorname{diam}\left(C_{l} \circ m K_{1}\right)=l / 2$, respectively. Now, $l / 2=$ $\lceil 2 n / 3\rceil=\lceil 4 m / 3\rceil$ and $l=2\lceil 4 m / 3\rceil$. Now, $2^{n}=m l=2 m\lceil 4 m / 3\rceil$, and we have $2^{n-1}=$ $m\lceil 4 m / 3\rceil$.

We discuss the value of $m$, as follows.

Case 1. $m=3 k$ where $k$ is a positive integer.

Then, $2^{n-1}=m\lceil 4 m / 3\rceil=4 m k=12 k^{2}$. Now, we obtain $2^{n-3}=3 k^{2}$, a contradiction.

Case 2. $m=3 k+1$ where $k$ is a positive integer.

Then, $2^{n-1}=m\lceil 4 m / 3\rceil=(3 k+1)\lceil(12 k+4) / 3\rceil=(3 k+1)(4 k+2)$. So, we have $2^{n-2}=(3 k+1)(2 k+1)$. In this case, $(2 k+1) \mid 2^{n-2}$; hence, we have $k=0$ and $m=1$. This contradicts the fact that $m$ is even. 
Case 3. $m=3 k+2$ where $k$ is a positive integer.

Then, $2^{n-1}=m\lceil 4 m / 3\rceil=(3 k+2)\lceil(12 k+8) / 3\rceil=(3 k+2)(4 k+3)$. So, we have $2^{n-1}=(3 k+2)(4 k+3)$ and $(4 k+3) \mid 2^{n-1}$, a contradiction.

Now, suppose that $V Q_{n} \cong L\left(Q_{3}\right) \circ m K_{1}$, then the number of vertices of these graphs is same and we obtain $2^{n}=12 m$, so we have $2^{n-2}=3 m$ and $3 \mid 2^{n-2}$ where $n \geq 3$, a contradiction.

Theorem 2. $V Q_{n}$ is super- $\lambda$ for $n \neq 2$.

Proof. Since $V Q_{1}$ is a complete graph $K_{2}$, it is super- $\lambda$. If $n=2, V Q_{n}$ is a cycle $C_{4}$. Clearly, it is not super- $\lambda$. Suppose that $V Q_{n}$ is not super- $\lambda$ for $n \geq 3$. $V Q_{n}$ is edge-transitive $k$-regular graph where $k \geq 3$, so the theorem follows from Proposition 2(1).

In [19], Xu et al. proved that 1-restricted edge-connectivity of $V Q_{n}$ is $2 n-2$. Here, we present another proof of this result.

Theorem 3. $\lambda^{\prime}\left(V Q_{n}\right)=2 n-2$ for $n \geq 2$.

Proof. If $n=2$, then $V Q_{n}$ is a cycle $C_{4}$. Clearly, $\lambda^{\prime}\left(V Q_{n}\right)=2$. Thus, we may suppose that $n \geq 3$. $V Q_{n}$ is an edge-transitive $k$-regular graph where $k \geq 3$, so the theorem follows from Proposition 2(2).

In the following theorem, we show that every minimum edge-cut of $V Q_{n}$ for $n \geq 3$ and $n \neq 4$ isolates an edge.

Theorem 4. $V Q_{n}$ is super- $\lambda^{\prime}$ for $n \geq 3$ and $n \neq 4$.

Proof. Assume that $V Q_{n}$ is not super- $\lambda^{\prime}$ for $n \geq 3$ and $n \neq 4$. According to $V Q_{n}$ is edgetransitive and Proposition 2(4), we know that $V Q_{n}$ is not super- $\lambda^{\prime}$ if and only if $V Q_{n}$ is isomorphic to $Q_{3}$ or to an edge-transitive 4-regular graph of girth 4.

If $n=3, Q_{3}$ does not have cycle of length 5 but $V Q_{3}$ has, then $V Q_{3}$ is not isomorphic to $Q_{3}$.

If $n \geq 5$, since $V Q_{n}$ is edge-transitive $k$-regular graph where $k \geq 5$, then it implies that $V Q_{n}$ is super- $\lambda^{\prime}$ when $n \geq 5$.

We have accordingly proved the theorem.

In the following theorem, we prove that if we remove $4(n-2)$ edges from the $V Q_{n}$, then we will obtain a disconnected graph with at least two components containing cycle for $n \geq 3$.

Theorem 5. $\lambda_{c}\left(V Q_{n}\right)=4(n-2)$ for $n \geq 3$.

Proof. Since $V Q_{n}$ has at least 8 vertices for $n \geq 3$, it is not isomorphic to $K_{4}, K_{5}$ or $K_{3,3}$. According to the fact that $V Q_{n}$ has girth 4 , it follows that $\lambda_{c}\left(V Q_{n}\right)=4(n-2)$ by Proposition 2(3).

The following theorem shows that every minimum cyclic edge-cut of $V Q_{n}$ isolates a shortest cycle for $n \geq 3$.

Theorem 6. $V Q_{n}$ is super $-\lambda_{c}$ for $n \geq 3$.

Proof. Since $V Q_{n}$ is an edge-transitive $n$-regular graph of girth $g=4$ with $2^{n}$ vertices. By the definition of $V Q_{n}$, we obtain that $V Q_{n}$ is not isomorphic to $K_{4}, K_{5}$ or $K_{3,3}$. If $n=3$, then it is obvious that $V Q_{n}$ is super- $\lambda_{c}$.

It is assumed that $V Q_{n}$ is not super- $\lambda_{c}$ for $n \geq 4$. So if $n=4$, then $V Q_{n}$ is edgetransitive 4-regular graph. According to Proposition 3(3), it follows that $\Gamma$ is isomorphic 
to $Q_{4}$ (the 4-cube), $K_{5.5}-5 K_{2}, C_{n} \circ 2 K_{1}(n \geq 4)$, or $\mathcal{D}(H)$, for some arc-transitive 4-regular graph $H$. Since $V Q_{4}$ has odd cycle of length 5 and 16 vertices, it is not difficult to check that $V Q_{4}$ is not isomorphic to $Q_{4}, K_{5.5}-5 K_{2}$, or $\mathcal{D}(H)$, for some 4-regular arc-transitive graph $H$. Furthermore, if $V Q_{n} \cong C_{l} \circ 2 K_{1}$ for $n=4$ and $l \geq 4$, then $\left.\mid V\left(C_{l} \circ 2 K_{1}\right]\right)|=| V\left(V Q_{n}\right) \mid$, it follows that $2 l=2^{4}=16$ and $l=8$. However, in this case, $\operatorname{diam}\left(V Q_{n}\right)=\lceil 2 n / 3\rceil=3$ and $\operatorname{diam}\left(C_{l} \circ 2 K_{1}\right)=\lfloor l / 2\rfloor=4$ by Lemma 1 and Lemma 5. So, $V Q_{n}$ is not isomorphic to $C_{l} \circ 2 K_{1}$, a contradiction. Now, by Proposition 3(3) and (4), $V Q_{5}$ or $V Q_{6}$ has 12 or 16 vertices, a contradiction.

Thus, we conclude that $V Q_{n}$ is super- $\lambda_{c}$ for $n \geq 3$.

The next theorem shows that every minimum vertex-cut of $V Q_{n}$ isolates an edge for $n \geq 9$.

Theorem 7. $V Q_{n}$ is super- $\kappa^{\prime}$ for $n \geq 9$.

Proof. By Lemma 2, we know that $\kappa^{(2)}\left(V Q_{n}\right)>\kappa^{(1)}\left(V Q_{n}\right)$ for $n \geq 9$. It is assumed that $V Q_{n}$ is not super- $\kappa^{\prime}$ for $n \geq 9$. Hence, there is an 1-restricted vertex-cut $S$ and $|S|=\kappa^{(1)}\left(V Q_{n}\right)$, but the cut is not the neighborhood of any edge. That is, $S$ is also a two-restricted vertex-cut and $|S| \geq \kappa^{(2)}\left(V Q_{n}\right)$. So, we have $|S|>\kappa^{(1)}\left(V Q_{n}\right)$, and this leads to a contradiction.

\section{Concluding Remarks}

In order to better measure the performance of the network, the concepts of restricted connectivity, restricted edge-connectivity, and cyclic edge-connectivity have been successively proposed. The research in this paper is based on these concepts and uses some properties of vertex-transitive and edge-transitive graph to get the main result that the varietal hypercube is super restricted vertex-connected, super restricted edge-connected, and super cyclically edge-connected. Moreover, the cyclic edge-connectivity of $V Q_{n}$ is $4(n-2)$ for $n \geq 3$. In the future, we will continue to research the other properties of the varietal hypercube.

Author Contributions: Z.Y.: Conceptualization, Investigation, Validation, Writing-original draft. L.X.: Conceptualization, Methodology, Investigation, Supervision, Validation, Writing-review \& editing. S.Y.: Investigation, Validation. L.G.: Investigation, Validation. All authors have read and agreed to the published version of the manuscript.

Funding: This work is supported by Natural Science Foundation of Fujian Province, China (Nos. 2021J01860,2019J01857 ), National Natural Science Foundation of China (Nos. 11301217,11961051) and Digital Fujian Big Data Modeling and Intelligent Computing Institute Open Fund Project.

Institutional Review Board Statement: Not applicable.

Informed Consent Statement: Not applicable.

Data Availability Statement: Not applicable.

Conflicts of Interest: The authors declare that they have no financial and personal relationships with other people or organizations that can inappropriately influence their work, there is no professional or other personal interest of any nature or kind in any product, service and/or company that could be construed as influencing the position presented in, or the review of, the manuscript entitle.

\section{References}

1. Elwekeil, M.; Abdalzaher, M.S.; Seddik, K. Prolonging smart grid network lifetime through optimising number of sensor nodes and packet length. IET Commun. 2019, 13, 2478-2484. [CrossRef]

2. Xu, J.-M. Combinatorial Theory in Networks; Academic Press: Beijing, China, 2013; pp. 3-27.

3. Li, X.-J.; Zeng, X.-Q.; Xu, J.-M. Note on reliability evaluation of arrangement graphs. Appl. Math. Comput. 2022, $418,126845$. [CrossRef]

4. Harary, F. Conditional connectivity. Networks 1983, 13, 347-357. [CrossRef] 
5. Esfahanian, A.H.; Hakimi, S.L. On computing a conditional edge-connectivity of a graph. Inf. Process. Lett. 1988, 27, 195-199. [CrossRef]

6. Chen, Y.-C.; Tan, J.-J.; Hsu, L.-H.; Kao, S.-S. Super-connectivity and super-edge-connectivity for some interconnection networks. Appl. Math. Comput. 2003, 140, 245-254. [CrossRef]

7. Xu, J.-M.; Wang, J.-W.; Wang, W.-W. On super and restricted connectivity of some interconnection networks. Ars Combin. 2010, 94, $25-32$.

8. Zhou, J.-X.; Wu, Z.-L.; Yang, S.-C.; Yuan, K.-W. Symmetric property and reliability of balanced hypercube. IEEE Trans. Comput. 2014, 64, 876-881. [CrossRef]

9. Bollobás, B. Extremal Graph Theory; Academic: Cambridge, MA, USA, 1978; pp. 110-119.

10. Plummer, M.D. On the cyclic connectivity of planar graphs, Graph Theory and Applications; Springer: Berlin/Heidelberg, Germany, 1972; pp. 235-242.

11. Zhang, Z.; Wang, B. Super cyclically edge connected transitive graphs. J. Comb. Optim. 2011, 22, 549-562. [CrossRef]

12. Zhang, W.-Q. The cyclic edge-connectivity of strongly regular graphs. Graphs Comb. 2019, 35, 779-785. [CrossRef]

13. Wang, B.; Zhang, Z. On cyclic edge-connectivity of transitive graphs. Discret. Math. 2009, 309, 4555-4563. [CrossRef]

14. Zhou, J.-X. On g-extra connectivity of hypercube-like networks. J. Comput. Syst. Sci. 2017, 88, 208-219. [CrossRef]

15. Wang, Y.; Feng, Y.-Q.; Zhou, J.-X. Automorphism group of the varietal hypercube graph. Graphs Comb. 2017, 33, 1131-1137. [CrossRef]

16. Barpanda, N.K.; Jena, S.; Dash, R.K. Network reliability evaluation of fault tolerant varietal hypercube network. Int. J. Adv. Res. Comput. Sci. 2014, 5, 132-135.

17. Cheng, S.-Y.; Chuang, J.-H. Varietal hypercube-A new interconnection network topology for large scale multicomputer. In Proceedings of the 1994 International Conference on Parallel and Distributed Systems, Hsinchu, Taiwan, 19-21 December 1994; pp. 703-708.

18. Cao, J.; Li, X.; Xu, J.-M. Cycles and paths embedded in varietal hypercubes. J. Univ. Sci. Technol. China 2014, 44, 732-737.

19. Wang, J.-W.; Xu, J.-M. Reliability analysis of varietal hypercube networks. J. Univ. Sci. Technol. China 2009, 39, $1248-1252$.

20. Xiao, L.; Cao, J.; Xu, J.-M. Transitivity of varietal hypercube networks. Front. Math. China 2014, 9, 1401-1410. [CrossRef]

21. Du, D.-Z.; Hsu, D.F. Combinatorial Network Theory; Springer: London, UK, 1996; pp. 41-60.

22. Meng, J. Connectivity of vertex and edge transitive graphs. Discret. Appl. Math. 2003, 127, 601-613. [CrossRef]

23. Boesch, F.; Tindell, R. Circulants and their connectivities. J. Graph Theory 1984, 8, 487-499. [CrossRef]

24. Li, Q.-L.; Li, Q. Super edge connectivity properties of connected edge symmetric graphs. Networks 1999, 33, 157-159. [CrossRef]

25. Tian, Y.; Meng, J. On super restricted edge-connectivity of edge-transitive graphs. Discret. Math. 2010, 310, 2273-2279. [CrossRef]

26. Zhou, J.-X. Super restricted edge connectivity of regular edge-transitive graphs. Discret. Appl. Math. 2012, 160, 1248-1252. [CrossRef]

27. Zhou, J.-X. Atoms of cyclic edge connectivity in regular graphs. J. Comb. Optim. 2016, 31, 382-395. [CrossRef]

28. Zhou, J.-X.; Feng, Y.-Q. Super-cyclically edge-connected regular graphs. J. Comb. Optim. 2013, 26, 393-411. [CrossRef]

29. Bondy, J.A.; Murty, U.S.R. Graph Theory; Springer: Berlin/Heidelberg, Germany, 2008; p. 327.

30. Li, H.; Yang, W. Bounding the size of the subgraph induced by $m$ vertices and extra edge-connectivity of hypercubes. Discret. Appl. Math. 2013, 161, 2753-2757. [CrossRef]

31. Chang, X.; Ma, J.; Yang, D.-W. Symmetric property and reliability of locally twisted cubes. Discret. Appl. Math. 2021, 288, 257-269. [CrossRef]

32. Xu, J.-M.; Yang, C. Connectivity of lexicographic product and direct product of graphs. Ars Combin. 2013, 111, 3-12.

33. Hammack, R.H.; Imrich, W.; Klavžar, S. Handbook of Product Graphs; CRC Press: Boca Raton, FL, USA, 2011 ; pp. 56-59. 